\title{
Coarsening of Complex Microstructures Following Spinodal Decomposition
}

\author{
C.-L. Park ${ }^{\mathrm{a}}$, J. W. Gibbs ${ }^{\mathrm{b}}$, P. W. Voorhees ${ }^{\mathrm{b}}$, K. Thornton ${ }^{\mathrm{a}, *}$ \\ ${ }^{a}$ Department of Materials Science and Engineering, University of Michigan, \\ 2300 Hayward, Ann Arbor, MI 48109, USA

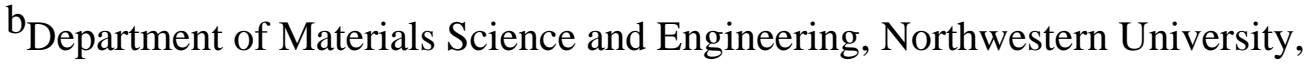 \\ 2220 Campus Drive, Evanston, IL 60201, USA
}

\begin{abstract}
Coarsening plays a pivotal role in materials engineering, but our understanding of the dynamics of coarsening in morphologically complex systems is still limited. In this paper, we examine the correlations between the interfacial velocity and interfacial morphologies, and then predict the evolution of mean curvature based on the correlations. Three simulated structures with varying volume fractions, two bicontinuous and one nonbicontinuous, are generated using the CahnHilliard equation. We find general correlations between interfacial velocity and mean curvature, as well as between interfacial velocity and the surface Laplacian of the mean curvature. Furthermore, we find that the probability of finding a patch of interface with a given normal velocity and the same local principal curvatures is described well by a Gaussian distribution, independent of the principal curvature values and the volume fractions of the structures. We also find that average interfacial velocity is described by a polynomial of the mean curvature and the net curvature. Based on this finding, we develop a semi-analytical approach to predicting the rate of change of the mean curvature, which determines the morphological evolution of complex microstructures.
\end{abstract}


*Corresponding author: Email address: kthorn@umich.edu, Tel: +17346151498 


\section{Introduction}

Coarsening is a phenomenon that is widely observed during thermal processing of many materials. It occurs during material processing of metallic alloys [1-4], polymers [5-8], bicontinuous nanoporous gold $[9,10]$ and semiconductors [11-13]. Following either spinodal decomposition or nucleation and growth, a two-phase system undergoes coarsening after the concentration of each phase approaches its equilibrium value. During this capillarity-driven phenomenon, solute diffuses from regions with high chemical potential to regions with low chemical potential as a result of the Gibbs-Thomson effect, which relates the chemical potentials to interfacial curvature. This diffusion process, in response to a thermodynamic driving force, reduces the total interfacial area within the microstructure to minimize the excess energy associated with interfaces.

Understanding the mechanisms of coarsening is important since the microstructure can undergo substantial changes in morphology and topology during coarsening. These changes in microstructure, in turn, directly influence the properties of the material after processing and during its utilization. For example, in the case of precipitation hardening of metallic alloys, the precipitates that nucleate in the solid solution undergo coarsening during the aging process. While the average size of the precipitates increases during coarsening, the number density of precipitates decreases, which enables dislocations to migrate more freely in the solid. As coarsening continues, the yield strength of the alloy decreases, resulting from the microstructural evolution [14]. In the case of nickel/yttria-stabilized zirconia (Ni/YSZ) cermet, which is a commonly used anode material in solid oxide fuel cells (SOFCs), the coarsening of Ni particles in the anode reduces the density of triple-phase boundaries, thus significantly diminishing the 
electrochemically active regions and degrading the electrochemical performance of SOFCs over time [15-17]. The mechanical properties of nanoporous gold structures can be linked to the topology of the bicontinuous structure that can evolve during coarsening $[18,19]$.

Coarsening of polydispersed spherical precipitates has been studied extensively in the past [2022]. In 1960s, Lifshitz and Slyozov [23], and Wagner [24] (LSW) determined analytically the growth rate of a particle, $d R / d t$, as a function of radius, $R$. Using the growth rate, they also determined the time-independent particle size distribution that is scaled by a time-dependent characteristic length scale of the system. The resulting LSW theory predicts $\langle R\rangle \sim t^{1 / 3}$. Even though the LSW theory is derived in the limit of zero volume fraction of the particle phase, in which the diffusion fields between particles do not overlap, it provides valuable insights into the self-similar evolution of a system of particles undergoing coarsening. Furthermore, this classical theory has served as a foundation for the subsequent development of more general theories that advanced the understanding of the dynamics of coarsening in materials with polydispersed precipitates (see comprehensive reviews [25, 26] and a book [27] for more information).

In contrast, the understanding of the dynamics of coarsening in materials with complex microstructures is still at an early stage. Deriving a theory of coarsening based solely on an analytical approach in these materials is extremely challenging because, unlike the simple spherical geometry that can be assumed in many theories of precipitate coarsening, the complex morphology and topology of the microstructure poses difficulties in solving the free-boundary problem describing the interfacial motion resulting from diffusion. For example, the coarsening of dendrites [28-30] has been studied by approximating the dendrite arms as a collection of cylinders [30] or cylinders with spherical caps at the end [29, 31]. However, such simple 
geometries do not fully capture the complex structures of dendrites, and therefore experimental and computational approaches are needed in elucidating the kinetics of interfacial motion during coarsening.

The ultimate goal of this work is to develop the theory of coarsening of complex microstructures. This requires a simplification of the dynamics because the complex morphology and topology of the structure introduces significant complications. Therefore, we begin with a simplified mathematical description of the process, based solely on the thermodynamic driving force from interfacial free energy and excluding other effects such as elastic stress and unequal mobilities in different phases. Such an approach gives important insights into the dynamics of coarsening processes in these morphologically complex systems.

In our previous effort, we examined the morphological evolution of a bicontinuous structure undergoing coarsening via nonconserved dynamics [32]. We observed that, while the majority of interfaces undergo flattening, some regions of interfaces become more highly curved as they approach pinching (a topological singularity). Furthermore, while the interfacial velocity is determined by the local mean curvature in nonconserved dynamics, the evolution of mean curvature is dependent on both the local curvature and the curvatures of the nearby interfaces since they can interact diffusionally.

Building upon this previous work, we here examine the coarsening of three complex structures following spinodal decomposition, in which the evolution of interfaces is due to the interfacialenergy-driven bulk diffusion. We first elucidate the dynamics of coarsening by examining the correlation between interfacial velocity and static interfacial properties, namely the mean curvature and the surface Laplacian of the mean curvature. In situations where the interfacial 
motion is dictated solely by the local geometry of the surface, such as grain growth [33, 34] and antiphase domain boundary motion [35], the exact relationship between the interfacial velocity and local curvatures can be determined. However, such a relationship cannot be established in a material system undergoing coarsening via bulk diffusion due to the long-range diffusional interactions. Therefore, we examine the statistical correlation (not a functional relationship) between the interfacial velocity and the mean curvature, as well as the correlation between the interfacial velocity and the surface Laplacian of the mean curvature. In addition, we explore the distribution of velocities of interfaces with given principal curvatures. Based on the information gathered from analysis of the interfacial velocity during coarsening, we develop a semianalytical approach to predicting the rate of change of the mean curvature, which is then verified against the simulation results.

\section{Computational Methods}

\subsection{Preparation of Microstructural Data}

The phase-field method is a computational technique for simulating phase transformations [3638]. The phase-field method is based on the diffuse-interface theory, where the value of an order parameter, , smoothly varies across an interfacial region with a finite thickness from a value representing one phase to another value representing a different phase. The diffuse-interface approach eliminates the need to explicitly track the location of interfaces since this information is embedded in , which is evolved based on the governing phase-field equation.

The governing equation employed in our simulation is the Cahn-Hilliard equation $[39,40]$ 


$$
\frac{\partial}{\partial t}=\nabla \cdot L \nabla\left(\begin{array}{ll}
\frac{\partial f}{\partial} & { }^{2} \nabla^{2}
\end{array}\right)
$$

where $L$ and $\epsilon$ are the mobility and the gradient energy coefficients, respectively. While $L$ can, in general, have dependence on and may vary spatially, we take it to be constant in this work for simplicity. The bulk free energy, $f($ ), is assumed to be a double-well potential,

$$
f()=\frac{W}{4}{ }^{2}(1 \quad)^{2}
$$

with two minima at $=0$ and $=1$ (the equilibrium values of the two phases), and the height of the double-well potential is controlled by a well-height parameter, $W$. Note that the term $\partial f / \partial \quad{ }^{2} \nabla^{2}$ is the chemical potential. The simulations are performed with a dimensionless form of the Cahn-Hilliard equation, where we define the dimensionless variables such that $x=\tilde{x} / l$, where $l$ is the scaling length, and $t=\tilde{t} /$, where is the associated time scale and the tilde indicates the dimensional variables. We select $l$ such that the grid spacings are

$$
x=y=z=1.0 \text { and } \quad \text { such that } L=\tilde{L} \quad / l^{2} \tilde{W}=1.0, \text { where } \tilde{L} \text { is the dimensional mobility }
$$

coefficient and $\tilde{W}$ is the dimensional well-height parameter. A time step of $t=0.05$ is employed. A computational domain size of $1024 \times 1024 \times 1024$ is chosen to generate sufficient statistics for accurate analyses of interfacial morphologies. In the phase-field method, values of

${ }^{2}$ and $W$ determine the interfacial thickness, . . In order to ensure sufficient interfacial resolution, we use ${ }^{2}=0.2$ and $W=0.4$ to obtain $=4.0$, which results in the interfacial region (defined by $\quad$ in the range 0.1 to 0.9 ) being approximately four-grid-point wide.

In order to examine the influence of overall morphologies on the dynamics of coarsening, we 
performed three separate simulations with different initial conditions that result in different volume fractions of the phases. The three initial conditions consist of order parameters with average values ${ }_{1}=0.5,,_{2}=0.4$, and ${ }_{3}=0.3$, each with a random noise of amplitude 0.1 . These initial conditions result in bicontinuous structures with 50:50 and 40:60 volume fractions of the phases, and a nonbicontinuous structure with 30:70 volume fractions of the phases. Since the Cahn-Hilliard equation is conserved, the volume fractions of the phases remain constant throughout their evolution.

The three structures were evolved for a dimensionless simulation time of $t_{0}=65,000$, which is sufficient to produce self-similar structures for the 50:50 and 40:60 volume fractions [41]. Hereafter, these simulated structures are labeled as 50:50, 40:60, and 30:70 Cahn-Hilliard (CH) structures, respectively. As a note, the self-similar morphology of the structure with the 30:70 $\mathrm{CH}$ structure should be a polydisperse distribution of spherical particles, as suggested by [41]. Therefore, the 30:70 $\mathrm{CH}$ structure is not self-similar, but rather evolving toward one consisting of spherical particles. Fig. 1 shows cubic portions of the three $\mathrm{CH}$ structures, each with side length $6 S_{v}{ }^{1}$, where $S_{v}{ }^{1}$ is the inverse of surface area per unit volume, which is employed as the characteristic length scale.
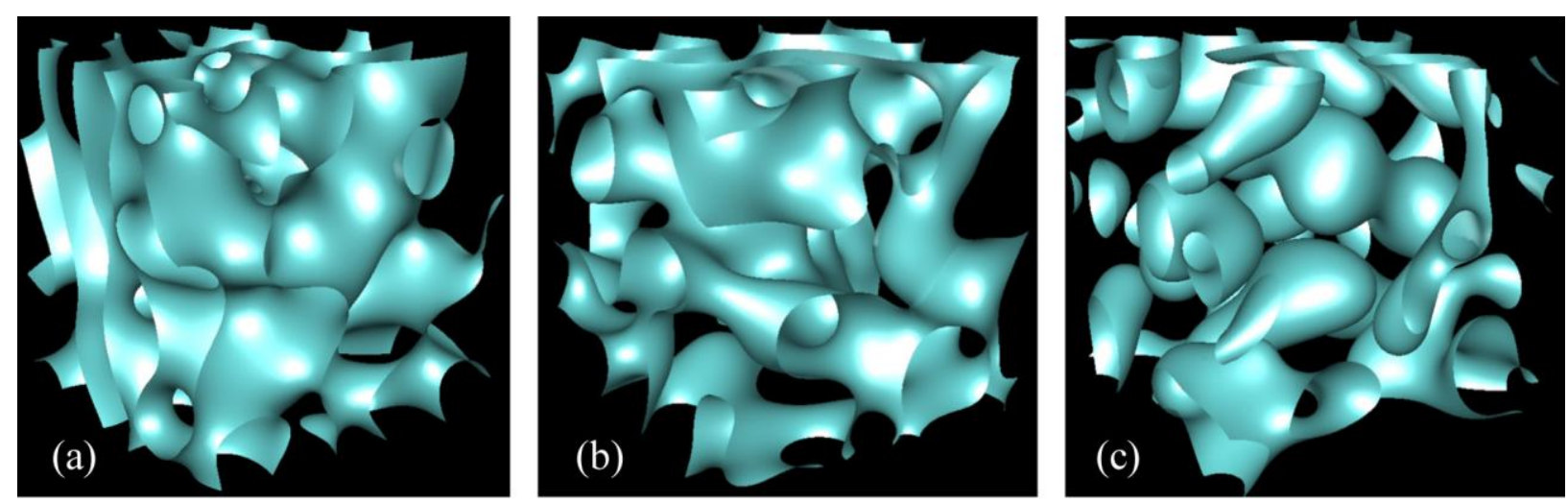

Figure 1. Morphologies of (a) the 50:50 CH structure, (b) the 40:60 CH structure, and (c) the 
30:70 CH structure at the reference simulation time of $t_{0}=65,000$. The structures in (a) and (b) are bicontinuous, while that in (c) is not bicontinuous. The morphologies shown are in cubic domains with side lengths six times their respective characteristic length scales, $S_{v}{ }^{1}$.

\subsection{Calculation of Interfacial Quantities}

Interfacial quantities can be classified into three categories: static local properties, static nonlocal properties, and dynamic quantities. The static local properties include the mean curvature, $H$, the Gaussian curvature, $K$, as well as the two principal curvatures, ${ }_{1}=H \quad \sqrt{H^{2}} K$ and

$$
{ }_{2}=H+\sqrt{H^{2} \quad K} \text {. We also evaluate the net curvature, } d=\sqrt{\left(\begin{array}{l}
2 \\
1
\end{array}+\begin{array}{l}
2 \\
2
\end{array}\right) / 2} \text {, which measures the }
$$

overall "bend" of the interface and reduces to $1 / R$ in spherical geometry [42]. The static nonlocal property we examine is the surface Laplacian of the mean curvature, ${ }_{S} H$, which describes the local variation of $H$ (but not mass transfer in this bulk-diffusion-limited process). The three dynamic quantities we examine are interfacial velocity, $v$, the rate of change of the mean curvature, $D H / D t$, and the surface Laplacian of interfacial velocity, ${ }_{s} v$. These dynamic quantities all appear in the expression for $D H / D t$ based on differential geometry,

$$
\frac{D H}{D t}=\left(\begin{array}{ll}
2 H^{2} & K
\end{array}\right) v \frac{1}{2}\left(v_{, 11}+v_{, 22}\right)
$$

where $v_{, 11}$ and $v_{, 22}$ are the second derivative of interfacial velocity along the principal directions (defined such that the two curvatures are maximum and minimum) and their sum is equal to the surface Laplacian of interfacial velocity, $s_{s} v[43]$.

In order to accurately calculate these interfacial quantities, we apply the level-set smoothing method [44]. The mean and Gaussian curvatures are calculated first, and other curvatures are 
then computed using the values of $H$ and $K$. The nonlocal static property, ${ }_{S} H$, is calculated using

$$
{ }_{s} H=H \quad 2 H \frac{\partial H}{\partial n} \quad \frac{\partial^{2} H}{\partial n^{2}}
$$

where $H$ is the Laplacian of $H$, and $H / n$ and ${ }^{2} H / n^{2}$ are the first and second derivative of $H$ with respect to the normal coordinate, respectively [45]. The interfacial velocity is calculated using the level-set method [46]. Based on this velocity, $D H / D t$, the Lagrangian derivative, is evaluated with respect to the coordinates moving with the interface. We use the advective method to calculate the Lagrangian derivative, as described in [44]. The surface Laplacian of interfacial velocity, ${ }_{s} v$, is calculated in the same manner as ${ }_{S} H$. All interfacial quantities presented in this paper are scaled by the characteristic length scale, $S_{v}{ }^{1}$, and the corresponding time scale, $S_{v}{ }^{1} /\left(d S_{v}{ }^{1} / d t\right)$, where $S_{v}{ }^{1}$ is calculated for the entire simulation volume for each structure.

\section{Procedures for Analysis}

This section briefly explains the various techniques employed to analyze the morphologies and their dynamics of interfaces in complex structures.

\subsection{Analysis of the Overall Morphologies and Interfacial Dynamics Using a Statistical} Approach

Statistical analysis reveals the global features of the morphology and the general relationships between interfacial quantities that govern the dynamics of coarsening. The characterization 
technique used in statistical analysis is the probability distribution as a function of various interfacial quantities. All of the probability distributions are calculated by examining each structure in its entirety. The probability distributions employed in this paper are described below.

The overall morphologies of the three structures are statistically characterized by the interfacial shape distributions (ISDs). The ISD is the probability distribution of finding a patch of interface with a given pair of principal curvatures, ${ }_{1}$ and ${ }_{2}$. The ISD can be mathematically represented as

$$
P_{\mathrm{ISD}}\left(\begin{array}{ll}
1, & 2
\end{array}\right)=\frac{A_{\mathrm{ISD}}\left(\begin{array}{ll}
1, & 2
\end{array}\right)}{A_{T}}
$$

where $A_{\mathrm{ISD}}\left(\begin{array}{ll}1 & 2\end{array}\right)$ is the total area of interfaces with a given pair of ${ }_{1}$ and ${ }_{2}$, and $A_{T}$ is the total interfacial area of the structure. $A_{\mathrm{ISD}}\left(\begin{array}{ll}1, & 2\end{array}\right)$ is numerically evaluated by summing interfacial areas that have curvatures within the range of ${ }_{1}^{i} \pm \quad / 2$ and ${ }_{2}^{i} \pm \quad / 2$, where ${ }_{1}^{i}$ and $\quad{ }_{2}^{i}$ are the center values of the $i$-th bin, and is the bin size in both $\quad_{1}$ and ${ }_{2}$ coordinates. We take to be 0.02 . All probability distributions presented in this paper are numerically evaluated in the same manner. Fig. 2 shows the graphical representation of the various interfacial shapes in the principal curvature space (also referred to as the ISD map). In the ISD map, regions 1 and 4 represent elliptic shapes, while regions 2 and 3 represent hyperbolic (saddle-like) shapes. In particular, when the mean curvature is zero, the local morphology of the interface becomes a perfectly symmetrical saddle shape. 


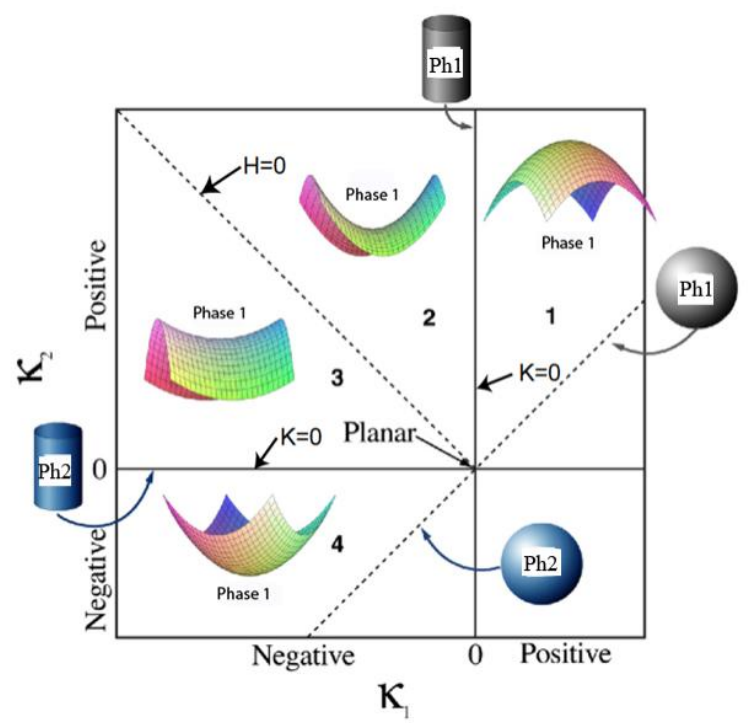

Figure 2. Graphical representation of interfacial shapes in the principal curvature space where the interfacial shape distribution is defined. Labels ' $\mathrm{Ph} 1$ ' and ' $\mathrm{Ph} 2$ ' represent the two phases in the system. Modified from Ref. [47].

The statistical relationship between the interfacial velocity and the local mean curvature is examined through the probability distribution, $P_{1}(H, v)$, defined as

$$
P_{1}(H, v)=\frac{A_{1}(H, v)}{A_{T}}
$$

where $A_{1}(H, v)$ is the total area of interfaces with given values of $H$ and $v$. The correlation between the interfacial velocity and the surface Laplacian of the mean curvature is also examined through the probability distribution, $P_{2}\left({ }_{s} H, v\right)$, defined as

$$
P_{2}\left({ }_{S} H, v\right)=\frac{A_{2}\left({ }_{S} H, v\right)}{A_{T}}
$$

where $A_{2}\left({ }_{s} H, v\right)$ is the total area of interfaces with given values of ${ }_{S} H$ and $v$. In addition, 
the correlation between the surface Laplacian of the mean curvature and the mean curvature is also examined through the probability distribution, $P_{3}\left(H,{ }_{S} H\right)$, defined as

$$
P_{3}\left(H,{ }_{S} H\right)=\frac{A_{3}\left(H,{ }_{S} H\right)}{A_{T}}
$$

where $A_{3}\left(H,{ }_{S} H\right)$ is the total area of interfaces with given values of $H$ and ${ }_{S} H$.

The distribution of normal velocities of interfaces with the same principal curvatures is examined through the probability distribution, $P_{4}\left({ }_{1}, 2, v\right)$, defined as

$$
P_{4}(1,2, v)=\frac{A_{4}(1,2, v)}{A_{\mathrm{ISD}}\left(\begin{array}{ll}
1, & 2
\end{array}\right)}
$$

where $A_{4}\left({ }_{1},{ }_{2}, v\right)$ is the total area of interfaces with given values of ${ }_{1},{ }_{2}$, and $v$.

In an effort to predict the rate of change of the mean curvature, we calculate the area-weighted averages of interfacial velocity, $\langle v\rangle_{H, d}$, the surface Laplacian of interfacial velocity, $\left\langle{ }_{S} v\right\rangle_{H, d}$, and the rate of change of the mean curvature, $\langle D H / D t\rangle_{H, d}$, of all interfaces with given values of $H$ and $d$. For example, $\langle v\rangle_{H, d}$ is evaluated as

$$
\langle v\rangle_{H, d}=\frac{A^{*}(H, d)}{A_{H, d}(H, d)}
$$

where $A^{*}(H, d)$ is the sum of the product of an area of an interfacial patch and its velocity at 
interfaces with given values of $H$ and $d$, and $A_{H, d}(H, d)$ is the total area of interfaces with given values of $H$ and $d$. Other averaged quantities, $\left\langle{ }_{S} v\right\rangle_{H, d}$ and $\langle D H / D t\rangle_{H, d}$, are calculated in the same manner.

\subsection{Analysis of Local Morphologies and Interfacial Dynamics}

To gain insights into the local morphological characteristics and evolution of the three $\mathrm{CH}$ structures, we visualize the interfacial quantities with shades of colors on the isosurfaces of the structure. For this analysis, we examine a cubic region of side length $6 S_{v}{ }^{1}$ from each of the $\mathrm{CH}$ structures. The relationship between the interfacial velocity and local curvature is examined by comparing the isosurfaces colored by the values of $v$ and $H$. We use the negative of the mean curvature for easier comparison of the two isosurfaces because $v$ and $H$, in general, have same sign. In addition, the influence of the local variation of curvature on the interfacial velocity is investigated by comparing isosurfaces colored by the values of $v$ and ${ }_{S} H$.

\section{Results \& Discussion}

\subsection{Overall Morphologies and Interfacial Dynamics}

Fig. 3(a.1)-(c.1) shows the interfacial shape distributions of the three $\mathrm{CH}$ structures. These ISDs are consistent with those calculated by Kwon et al. [41]. The ISDs of the 50:50 and 40:60 CH structures, Fig. 3(a.1) and (b.1), show that the majority of the interfaces are saddled-shaped. The ISD of the 50:50 $\mathrm{CH}$ structure is symmetric about the $H=0$ line due to the equal volume fraction of the two phases. On the other hand, the ISD of the 40:60 $\mathrm{CH}$ structure has similar shape as that for the 50:50 $\mathrm{CH}$ structure, but is slightly shifted to the left of the $H=0$ line as a 
result of the unequal volume fraction. In contrast to the two bicontinuous structures, the ISD of the 30:70 $\mathrm{CH}$ structure, Fig. 3(c.1), shows that the majority of the interfaces are elliptic surfaces. However, 30:70 is not self-similar, unlike the other volume fractions, and thus the ISD is evolving with time.

The correlations between $v$ and $H$, as well as $v$ and ${ }_{S} H$, are examined with the corresponding probability contour maps for all three structures, as shown in Fig. 3(a.2)-(c.2) and (a.3)-(c.3), respectively. For ease of comparison, a probability contour map of $P_{1}(H, v)$ is plotted against $H$. All plot ranges are kept constant for each type of plots. In Fig. 3(c.2), a second probability contour map of $P_{1}(H, v)$ of the 30:70 $\mathrm{CH}$ structure with a wider $H$ range is inserted to show the entire distribution. The plots of $P_{1}(H, v)$ show that $v$ and $H$ are strongly correlated. However, it is clear that the value of $H$ does not set $v$; there is a dispersion of velocities for a given $H$, unlike the case of nonconserved (Allen-Cahn) dynamics where $v$ is proportional to $H$ [35]. In addition, similar correlations can be observed between $v$ and ${ }_{s} H$. Furthermore, $P_{1}(H, v)$ and $P_{2}\left({ }_{s} H, v\right)$ for each structure have similar shape, but over different ranges of $H$ and ${ }_{s} H$, that differ by approximately a factor of four. However, the distribution with respect to $H$ shows a shift toward a greater value of $H$ for the asymmetric 40:60 and 30:70 $\mathrm{CH}$ structures as a consequence of the unequal volume fraction. The white solid curve in each of the contour maps represents the average interfacial velocity, $\langle v\rangle_{H}$ or $\langle v\rangle_{S^{H}}$, for a given value of $H$ or ${ }_{s} H$, respectively, which confirms the general correlations between the interfacial quantities. We find that both $\langle v\rangle_{H}$ and $\langle v\rangle_{S^{H}}$ are a good fit to cubic polynomials (not shown because the fit 
overlaps the average values); the fit parameters are shown in the appendix. The apparent discrepancy between $\langle v\rangle_{s^{H}}$ and the local maximum of the $P_{2}\left({ }_{s} H, v\right)$ distributions at $\left|{ }_{s} H\right|$ greater than two for all volume fractions is the result of the asymmetry of $P_{2}\left({ }_{s} H, v\right)$, which is not visible with the range of color chosen to show the entire distribution. Such asymmetry is nearly absent in the $P_{1}(H, v)$ and thus $\langle v\rangle_{H}$ coincides with the local maximum of the distribution. 


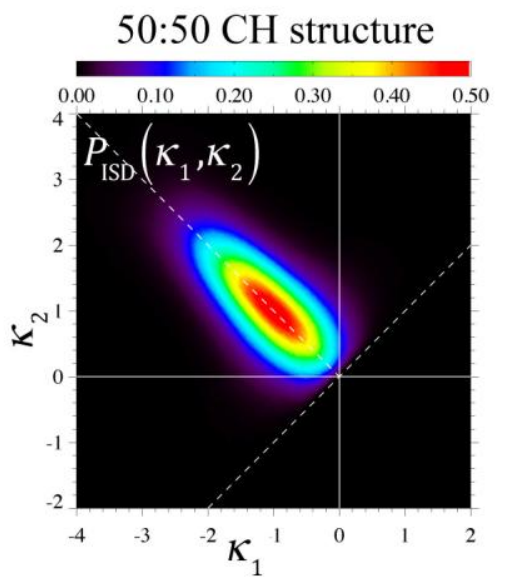

(a.1)

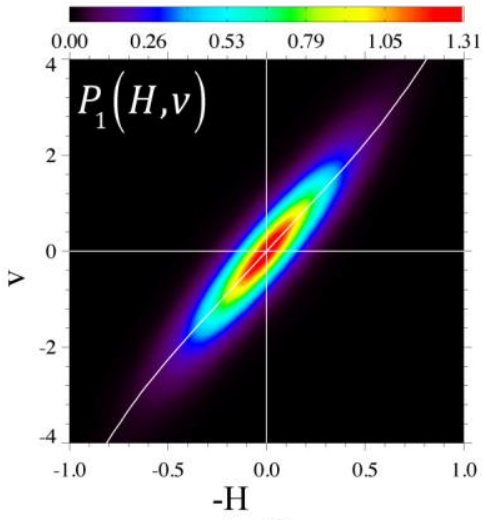

(a.2)

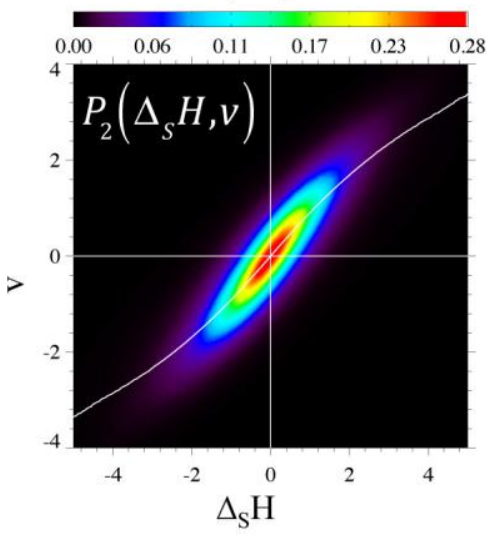

(a.3)

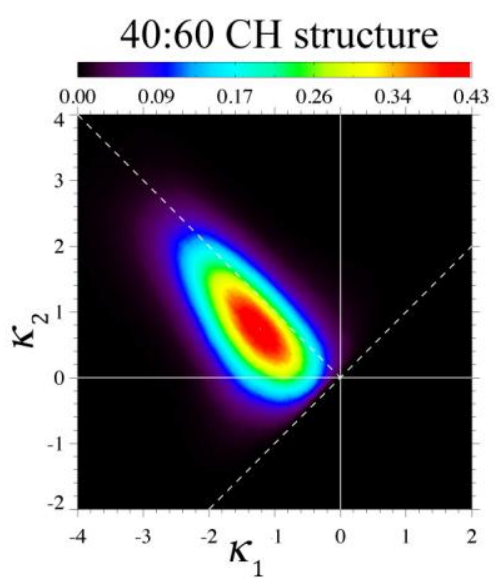

(b.1)

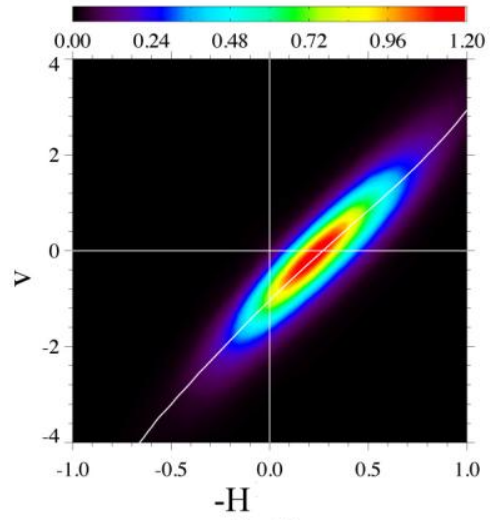

(b.2)

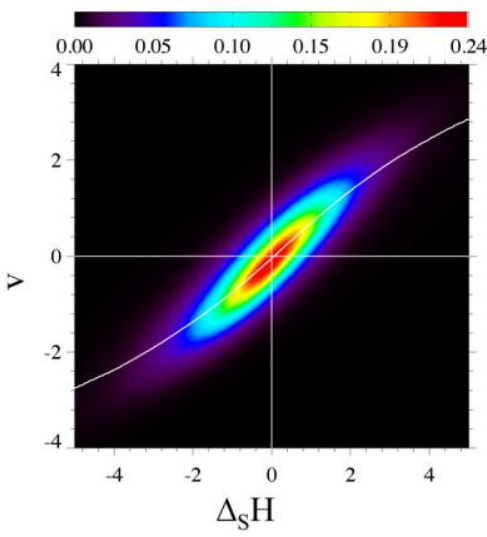

(b.3)

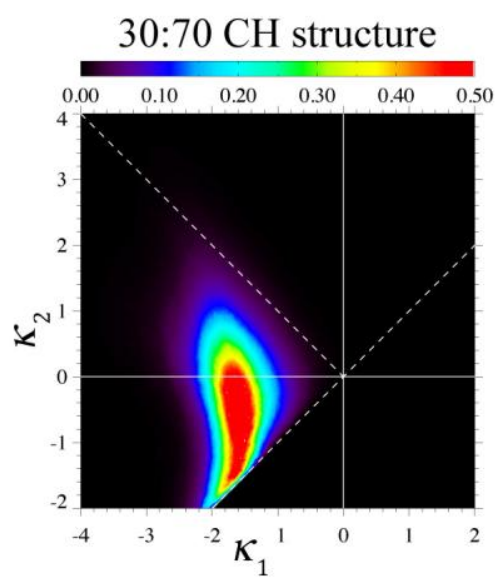

(c.1)

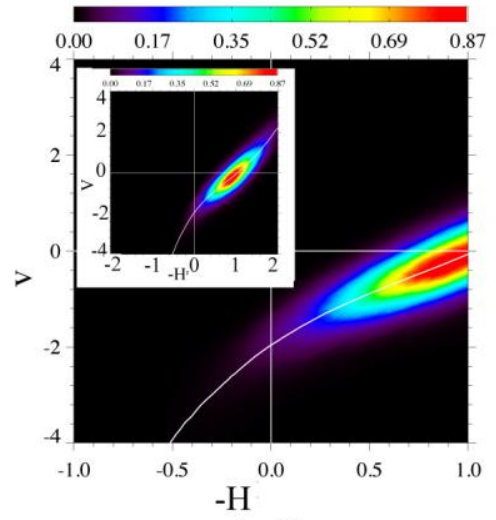

(c.2)

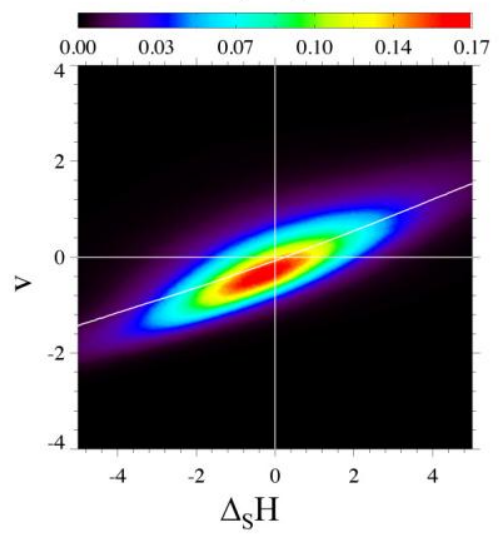

(c.3)

Figure 3. The interfacial shape distributions of (a.1) 50:50, (b.1) 40:60, and (c.1) $30: 70 \mathrm{CH}$ structure, along with the corresponding probability contour maps of $P_{1}(H, v)((\mathrm{a} .2)-(\mathrm{c} .2))$ and $P_{2}\left({ }_{s} H, v\right)(($ a.3)-(c.3)). The white solid curve in each of the contour map represents the average interfacial velocity, $\langle v\rangle_{H}$ and $\langle v\rangle_{{ }_{S}}$, for a given a value of $H$ and ${ }_{S} H$, respectively. 
Since the mean curvature determines the chemical potential at the interface, it is not surprising that $v$ and $H$ are correlated; interfaces with higher chemical potential are expected to evolve faster than those with lower chemical potentials on average. In fact, the correlation between $v$ and $H$ has also been observed in experimental microstructures that are free from the simplifying assumptions made in this paper. For example, a similar correlation between $v$ and $H$ was found in the solid-liquid dendritic mixtures of $\mathrm{Al}-\mathrm{Cu}$ alloy in which the diffusion of solute in the solid phase is negligible compared to that of the liquid phase [42]. It should be noted that the cubic term in the polynomial dependence of the average interfacial velocity, $\langle v\rangle_{H}$, on the mean curvature we observe is in direct contrast to the linear dependence of the interfacial velocity, $v$, on the mean curvature in nonconserved (Allen-Cahn) dynamics [35].

On the other hand, the physical origin of the correlation between $v$ and ${ }_{s} H$ is not obvious. In the case of interfacial energy driven surface diffusion [48], the two quantities are equal to within a constant of proportionality, and would thus yield straight lines in Fig. 3(a.3)-(c.3). In the CahnHilliard case, however, the interfacial velocity is determined by the normal gradient of the chemical potential at the interface, which is influenced by the chemical potentials of the nearby interfaces. Since the chemical potential at each interface is determined by its mean curvature (due to the Gibbs-Thomson effect), it is reasonable that ${ }_{s} H$, which measures a local variation of the mean curvature about a point on the interface, thus can be correlated with $v$. However, such a correlation has not been investigated in experiments to date.

Since the above results point to a possible correlation between $H$ and ${ }_{s} H$, we have examined $P_{3}\left(H,{ }_{s} H\right)$ for all three structures (not shown). The contour maps reveal that, on average, ${ }_{s} H$ 
has a negative correlation with $H$, but broader than other correlations we have identified. This correlation may be a result of the correlation between $v$ and ${ }_{s} H$, and $v$ and $H$ or, alternatively, it may be the result of a geometrically necessary variation in $H$ for the bicontinuous structure.

\subsection{Local Morphologies and Interfacial Dynamics}

The morphologies of the interfaces of the three $\mathrm{CH}$ structures colored by the negative of the mean curvature values are shown in Fig. 4(a.1)-(c.1). While the range on the color bars for Fig. 4(a.1) and (b.1) is symmetric and is equal to [-1, 1], the range for Fig. 4(c.1) is shifted and is set to $[0,2]$ to accommodate the substantial change in the range of the mean curvature in that structure as compared to the other two. All of the local morphologies exhibit features that are consistent with the ISDs presented in Fig. 3(a.1)-(c.1). The 50:50 CH structure appears to have an equal distribution of positive and negative mean curvature (consistent with the equal volume fractions of the two phases), while the $40: 60 \mathrm{CH}$ structure clearly has a larger proportion of interfaces with negative mean curvature, as observed in Fig. 4(a.1)-(b.1). The nonbicontinuous 30:70 $\mathrm{CH}$ structure possesses multiple isolated domains with near-spherical caps, as shown in Fig. 4(c.1). The fact that most of the surfaces of the isolated domains have negative mean curvature values indicates that these droplet-like interfaces enclose the minority phase (by the sign convention chosen). In addition, these isolated domains have larger $|H|$ than the saddleshaped interfaces in the 50:50 $\mathrm{CH}$ structure. An example of an isolated domain is noted by a red arrow in Fig. 4(c.1).

The local dynamics of coarsening is investigated by considering the interfacial velocities indicated by the shades of color on the interfaces, as shown in Fig. 4(a.2)-(c.2). The comparisons 
between Fig. 4(a.1)-(c.1) and (a.2)-(c.2) reveal a general correlation between interfacial velocity and mean curvature, which is consistent with the probability distributions $P_{1}(H, v)$ in Fig. 3(a.2c.2). In most regions, interfaces with large $|H|$ also have large $|v|$, as illustrated by the circled regions $\mathrm{A}$ through $\mathrm{C}$ in these figures. These regions include both saddle-shaped and elliptic surfaces, thus the correlation appears to hold for a wide range of interfacial morphologies without notable rules or exceptions. However, in the nonbicontinuous 30:70 $\mathrm{CH}$ structure, there are interfaces with $H \quad 0$ but have large $|v|$, as highlighted by the circled region D in Fig. 4(c.1) and (c.2). This is also consistent with the contour map of $P_{1}(H, v)$ for the 30:70 $\mathrm{CH}$ structure, where the center of the distribution is shifted from $v=0$ and $H=0$. This apparent discrepancy can be understood by considering the driving force of coarsening being the difference in the chemical potential of the interface with respect to the average (or mean-field) chemical potential of the surroundings. It has been suggested that the interfacial velocity may be modeled as $v \mu\left(\begin{array}{ll}H & H_{c}\end{array}\right)$, where $H_{c}$ is the critical mean curvature (which equals the average mean curvature) of the system [42]. In this case, the interfaces having $H \quad 0$ in the 30:70 $\mathrm{CH}$ structure, which has $H_{c}=1.1$, is expected to have a nonzero velocity. This is consistent with the observation from Fig. 4. However, as discussed in the previous subsection, there appears to be higher-order terms that exist when $v$ is modeled as a function of $H$. Nevertheless, this simple linear model may provide insights into the coarsening of complex microstructures by capturing the primary dependence of $v$ on $H$.

To examine the correlation between $v$ and ${ }_{s} H$ in detail, we compare the isosurfaces colored by the surface Laplacian of the mean curvature (Fig. 4 (a.3)-(c.3)) and the normal velocity (Fig. 4 
(a.2)-(c.2)). Consistent with $P_{2}\left({ }_{s} H, v\right)$, it is clear that $v$ and ${ }_{s} H$ have a positive correlation as displayed by the like-colors throughout the interfaces in all three structures, highlighted by the circled regions A through D in Fig. 4(a.2)-(c.2) and (a.3)-(c.3). This finding agrees with the contour map of $P_{2}\left({ }_{S} H, v\right)$, where the two quantities show a positive correlation and the centers of the distributions all lie at or near the origin. Again, we examined both saddle-shaped and elliptic surfaces, and no notable exceptions from the general correlation were found. 


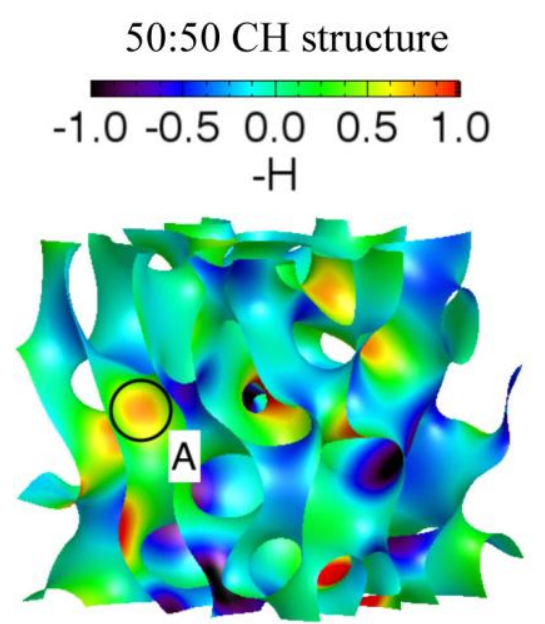

(a.1)
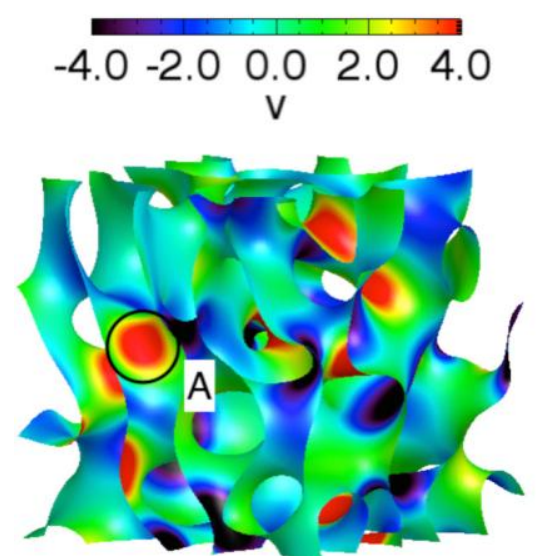

(a.2)

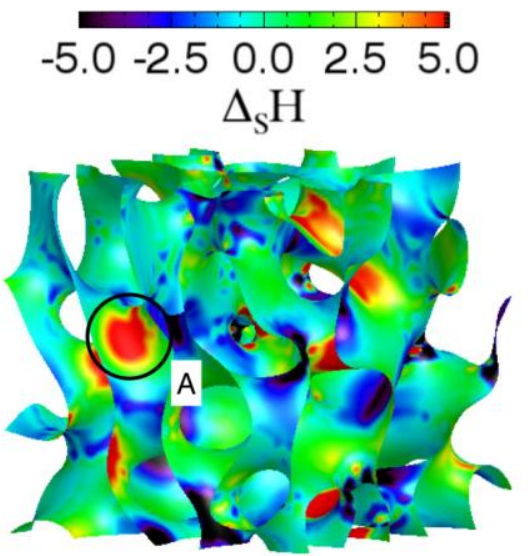

(a.3)

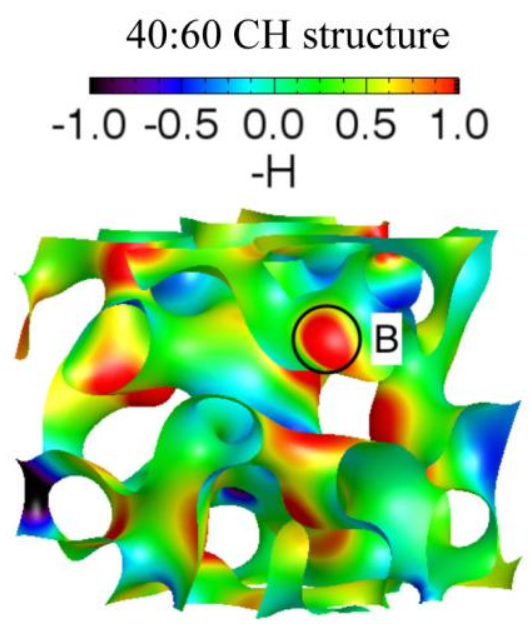

(b.1)
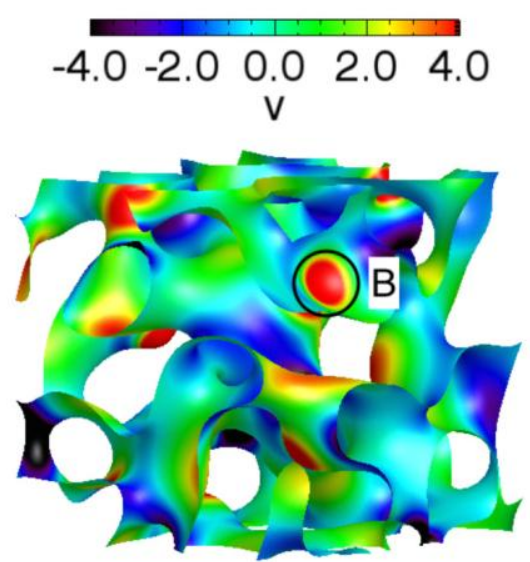

(b.2)

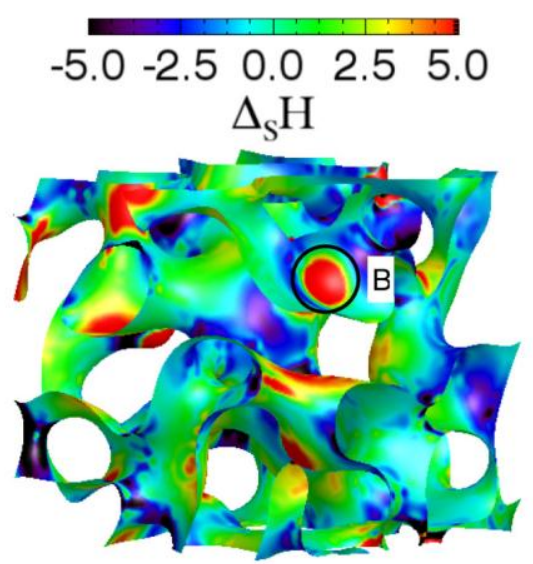

(b.3)

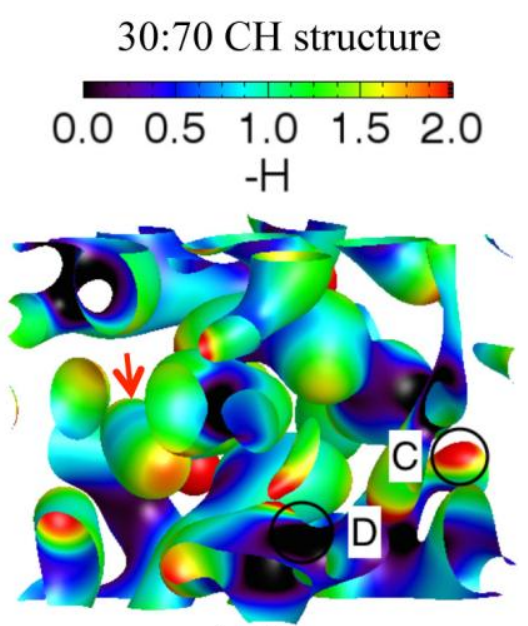

(c.1)
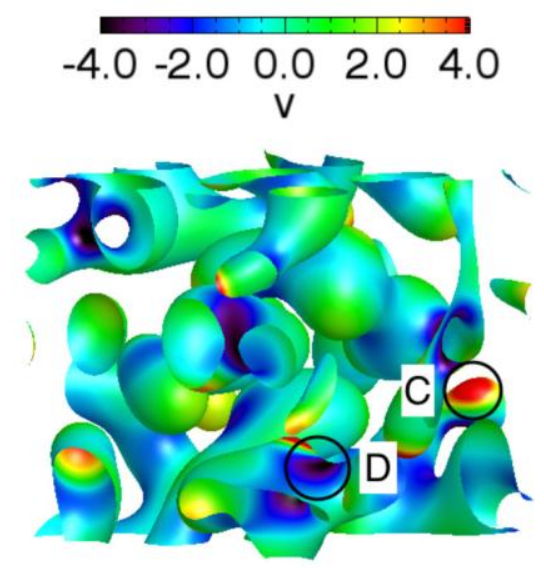

(c.2)

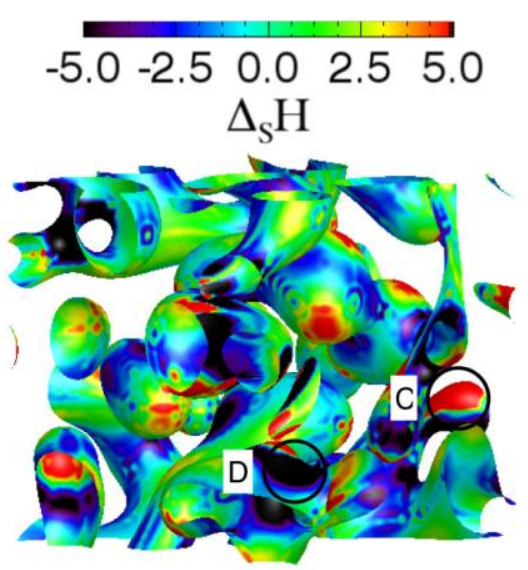

(c.3)

Figure 4. Morphologies of the $\mathrm{CH}$ structures with different volume fractions: (a.1)-(a.3) 50:50; (b.1)-(b.3) 40:60; and (c.1)-(c.3) 30:70, colored with (a.1)-(c.1) negative of the mean curvature, $H$; (a.2)-(c.2) interfacial velocity, $v$; and (a.3)-(c.3) the surface Laplacian of the mean 
curvature, ${ }_{S} H$. An example of an isolated domain in the $30: 70 \mathrm{CH}$ structure is noted by a red arrow in (c.1).

\subsection{Distribution of Interfacial Velocities}

The distribution of normal velocities of interfaces with the same principal curvatures, $P_{4}\left({ }_{1},{ }_{2}, v\right)$, is also examined. Figs. 5 and 6 show the ISD and $P_{4}\left({ }_{1},{ }_{2}, v\right)$ for the 50:50 and 30:70 $\mathrm{CH}$ structures, respectively. The principal curvature values at which $P_{4}\left({ }_{1},{ }_{2}, v\right)$ are plotted are shown on the ISD as solid blue circles with arrows pointing to the corresponding distributions (the actual ranges in the $i$-th bin are ${ }_{1}^{i} \pm 0.01$ and ${ }_{2}^{i} \pm 0.01$, as in the ISD calculation). For velocity, the bin size is taken to be $v=0.17$. Each distribution is then fitted with a Gaussian function; the fit parameters are shown in Table 1. In the table, ${ }_{1}$ and $\quad{ }_{2}$ are the curvature values chosen to plot $P_{4}\left({ }_{1},{ }_{2}, v\right),\langle v\rangle$ and ${ }_{V}$ are the average and the standard deviation of the Gaussian fit, respectively. To verify the goodness of fit, the quantiles from

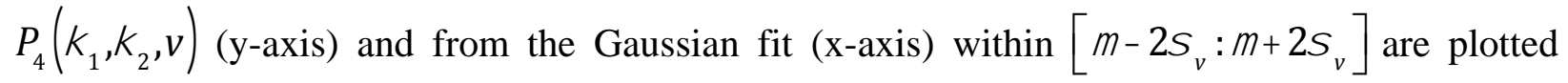
against each other and shown as a Q-Q (quantile-quantile) plot [49] adjacent to each histogram in Figs. 5 and 6. A q-quantile is generalization of quartile or percentile, where the number of partition of the range of a probability distribution (four for quartile and 100 for percentile, respectively) is equal to a value given by $q$. A $k$-th $q$-quantile of the velocity distribution is the value of the velocity that corresponds to the cumulative distribution equal to $k / q$ [49]. The sample quantiles from $P_{4}\left({ }_{1},{ }_{2}, v\right)$ are determined by sorting the velocity values of interfaces with the given set of principal curvatures. The theoretical quantiles from the Gaussian fit are determined analytically using the inverse error function. Since the majority of the Q-Q data 
points lie close to the $y=x$ line, it is evident that the Gaussian function fits the distributions very well, independent of the principal curvature values and the volume fraction of the system. As a note, the slight downward shift of the data points from the $y=x$ line at the left end of the Q-Q plot indicates that the Gaussian fit underestimates $P_{4}\left({ }_{1},{ }_{2}, v\right)$ at these (lower) velocity ranges. Similarly, the slight upward shift of the data points at the right end of the Q-Q plot indicates underestimation of $P_{4}\left({ }_{1}, 2, v\right)$ at the larger side of velocity range. These discrepancies between the sample and theoretical quantiles occur at the range of velocity distribution with relatively low statistics, which may be the origin of the deviation. The $40: 60 \mathrm{CH}$ structure also exhibited this behavior, but the plot is omitted due to space constraint.

The fact that a Gaussian function fits the velocity distribution across the wide range of curvatures is remarkable. However, it is even more surprising that it fits all volume fractions examined, the 50:50 and 40:60 $\mathrm{CH}$ structures that are evolving in a self-similar manner, and the $30: 70 \mathrm{CH}$ structure is in the process of breaking up into individual domains that consist of spherical particles. The latter is expected to be fundamentally different from the self-similar evolution of a bicontinuous structure. Furthermore, it is notable that, while $v$ has a wide range, ${ }_{v}$ is relatively constant across different curvature values for a given volume fraction. Specifically, ${ }_{V} \quad 0.5$ for the 50:50 $\mathrm{CH}$ structure and ${ }_{V} \quad 0.4$ for the $30: 70 \mathrm{CH}$ structure. This implies that velocities are randomly distributed about the average even though the microstructure is clearly not random. The origin of the Gaussian distribution has not been identified and requires further investigation. Since interfacial velocity is determined by long-range diffusion characterized by the diffusion distance, we plan to analyze the two-point correlation functions of $v$ and $H$ to further elucidate 
the dynamics of coarsening in complex microstructures, which will be a topic of a future publication.
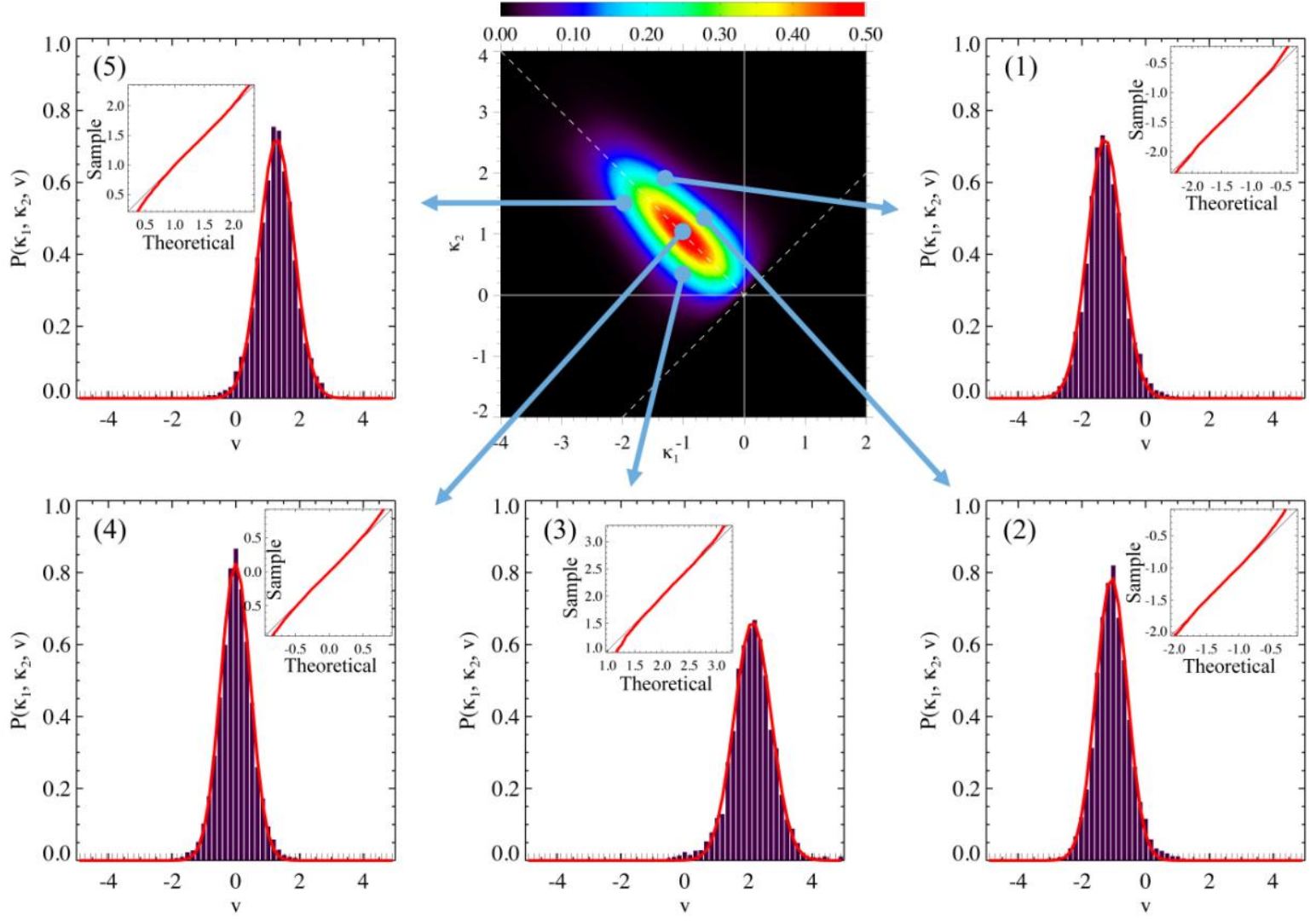

Figure 5. Probability distributions of interfacial velocities, $P_{4}\left({ }_{1},{ }_{2}, v\right)$, at various principal curvature values for the 50:50 $\mathrm{CH}$ structure. The principal curvature values at which $P_{4}\left({ }_{1},{ }_{2}, v\right)$ are plotted are shown on the ISD (top center) as solid blue circles with arrows pointing to the corresponding distributions. The Gaussian fit is represented as a solid red curve on each plot. To verify the goodness of fit, a Q-Q plot with the sample quantiles from $P_{4}\left({ }_{1},{ }_{2}, v\right)$ (y-axis) and the theoretical quantiles from the Gaussian fit (x-axis) is plotted adjacent to each histogram. The numbers marked on the top left corner of the plots correspond to the plot numbers in Table 1. 

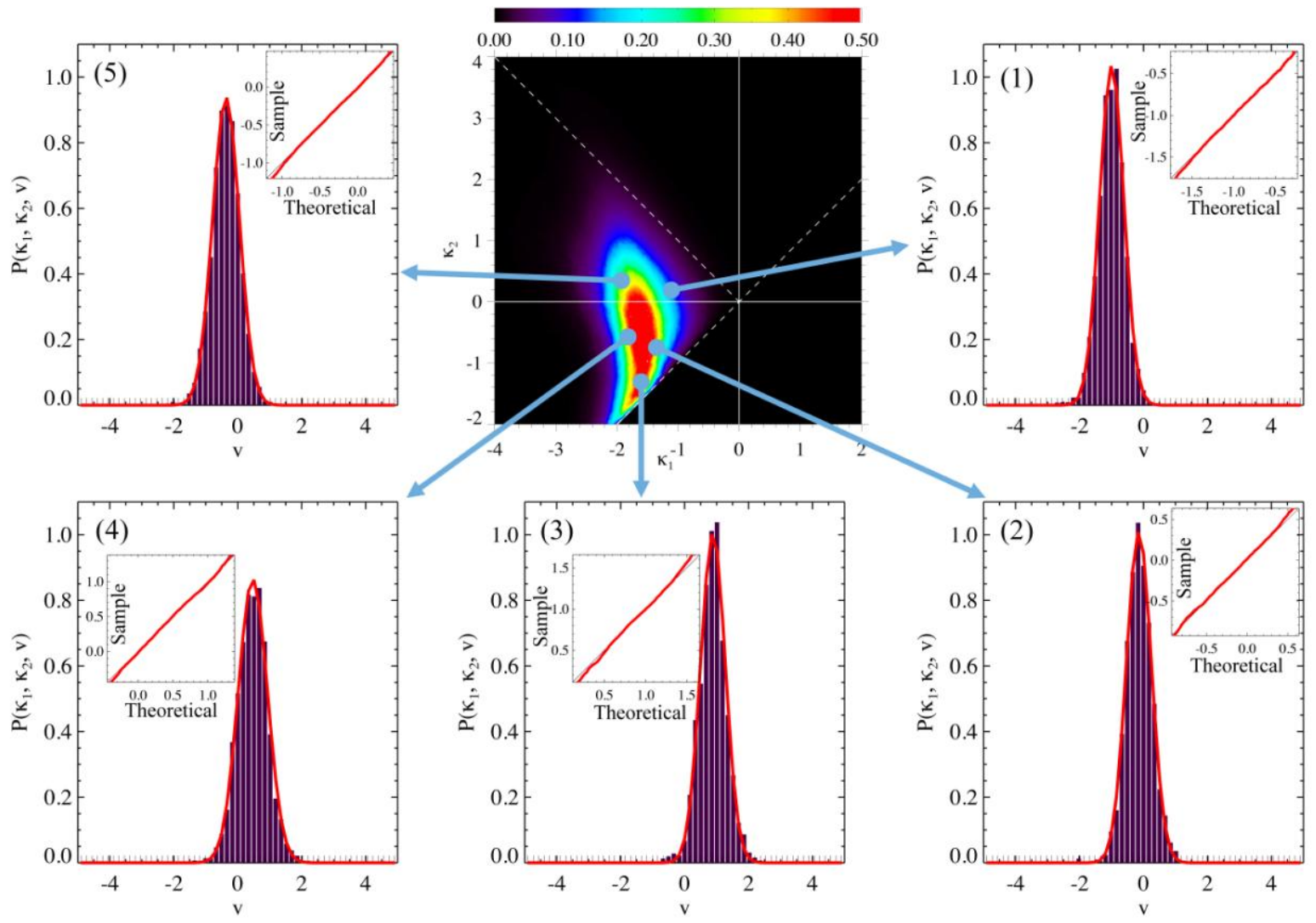

Figure 6. Probability distributions of interfacial velocities, $P_{4}\left({ }_{1},{ }_{2}, v\right)$, at various principal curvature values for the 30:70 $\mathrm{CH}$ structure. The principal curvature values at which $P_{4}\left({ }_{1},{ }_{2}, v\right)$ are plotted are shown on the ISD (top center) as solid blue circles with arrows pointing to the corresponding distributions. The Gaussian fit is represented as a solid red curve on each plot. To verify the goodness of fit, a Q-Q plot with the sample quantiles from $P_{4}\left({ }_{1},{ }_{2}, v\right)$ (y-axis) and the theoretical quantiles from the Gaussian fit (x-axis) is plotted adjacent to each histogram. The numbers marked on the top left corner of the plots correspond to the plot numbers in Table 1. 


\begin{tabular}{|c|c|r|r|r|r|}
\hline Structure & Plot \# & 1 & 2 & $\langle v\rangle$ & $V$ \\
\hline \hline \multirow{4}{*}{$\begin{array}{c}50: 50 \\
\text { CH }\end{array}$} & 1 & -1.5 & 2.0 & -1.29 & 0.54 \\
\cline { 2 - 6 } & 2 & -0.7 & 1.2 & -1.07 & 0.49 \\
\cline { 2 - 6 } Structure & 3 & -1.4 & 0.4 & 2.13 & 0.58 \\
\cline { 2 - 6 } & 4 & -1.3 & 1.3 & -0.01 & 0.47 \\
\cline { 2 - 6 } & 5 & -2.0 & 1.5 & 1.28 & 0.54 \\
\hline \multirow{4}{*}{$\begin{array}{c}\text { 30:70 } \\
\text { CH }\end{array}$} & 1 & -1.1 & 0.3 & -0.99 & 0.38 \\
\cline { 2 - 6 } Structure & 2 & -1.2 & -0.8 & -0.14 & 0.39 \\
\cline { 2 - 6 } & 3 & -1.6 & -1.5 & 0.89 & 0.39 \\
\cline { 2 - 6 } & 4 & -1.9 & -0.6 & 0.47 & 0.46 \\
\cline { 2 - 6 } & 5 & -1.9 & 0.3 & -0.37 & 0.42 \\
\hline
\end{tabular}

Table 1. Fit parameters of $P_{4}\left({ }_{1}, 2, v\right)$ presented in Figs. 5 and 6 . The plot numbers are the numbers indicated on the top left corner of each distribution plot. ${ }_{1}$ and ${ }_{2}$ are the curvature values chosen to plot $P_{4}\left({ }_{1},{ }_{2}, v\right)$, and $\langle v\rangle$ and ${ }_{V}$ are the average and the standard deviation of the Gaussian fit, respectively.

\subsection{Prediction of Curvature Evolution}

Having gained insights into the dynamics of interfaces during coarsening in complex structures, we now attempt to predict the evolution of the average curvature based on local morphological characteristics. As introduced in Sec. 2.2, the rate of change of the mean curvature, $D H / D t$, can be expressed as Eq. (3) based on differential geometry. Since $2 H^{2} \quad K=d^{2}$ and $v_{, 11}+v_{, 22}={ }_{s} v$, Eq. (3) can be re-written as

$$
\frac{D H}{D t}=d^{2} v \frac{1}{2}{ }_{s} v
$$

In our previous work on nonconserved dynamics, where $v=M H$ with $M$ being the proportionality constant [35], we were able to derive the expression for $D H / D t$ solely in terms 
of static interfacial properties, namely the local curvatures, $H$ and $K$, and the nonlocal interfacial property, ${ }_{s} H$ [32]. In conserved dynamics, even though $v$ is not proportional to the mean curvature, the statistical analysis in Sec. 4.1 demonstrated that the average interfacial velocity can be expressed in terms of a cubic polynomial of $H$. Based on this finding, we can derive an expression for the average rate of change of the mean curvature solely in terms of the local curvatures.

By averaging Eq. (11) over all interfaces with given values of $H$ and $d$, the average rate of change of the mean curvature, $\langle D H / D t\rangle_{H, d}$, with given values of $H$ and $d$ can be written as

$$
\left\langle\frac{D H}{D t}\right\rangle_{H, d}=d^{2}\langle v\rangle_{H, d} \frac{1}{2}\left\langle{ }_{S} v\right\rangle_{H, d}
$$

where $\langle v\rangle_{H, d}$ and $\left\langle{ }_{S} v\right\rangle_{H, d}$ are the average interfacial velocity and surface Laplacian of interfacial velocity, respectively, of all interfaces with given values of $H$ and $d$. The expressions for $\langle v\rangle_{H, d}$ and $\left\langle{ }_{S} v\right\rangle_{H, d}$ in terms of $H$ and $d$ are numerically determined through curve fitting the simulation data. We then substitute two expressions into Eq. (12) to express $\langle D H / D t\rangle_{H, d}$ in terms of the local curvatures. The curvatures $H$ and $d$ are chosen to express $\langle v\rangle_{H, d}$ and $\left\langle{ }_{S} v\right\rangle_{H, d}$ since $H$ alone cannot fully describe the local morphology, and thus we require a second parameter. The net curvature, $d$, is chosen since, unlike the Gaussian curvature, it has the same unit as $H$ and it has an intuitive interpretation as the distance from the origin on the ISD map, representing a measure of the curvature of the interface. 

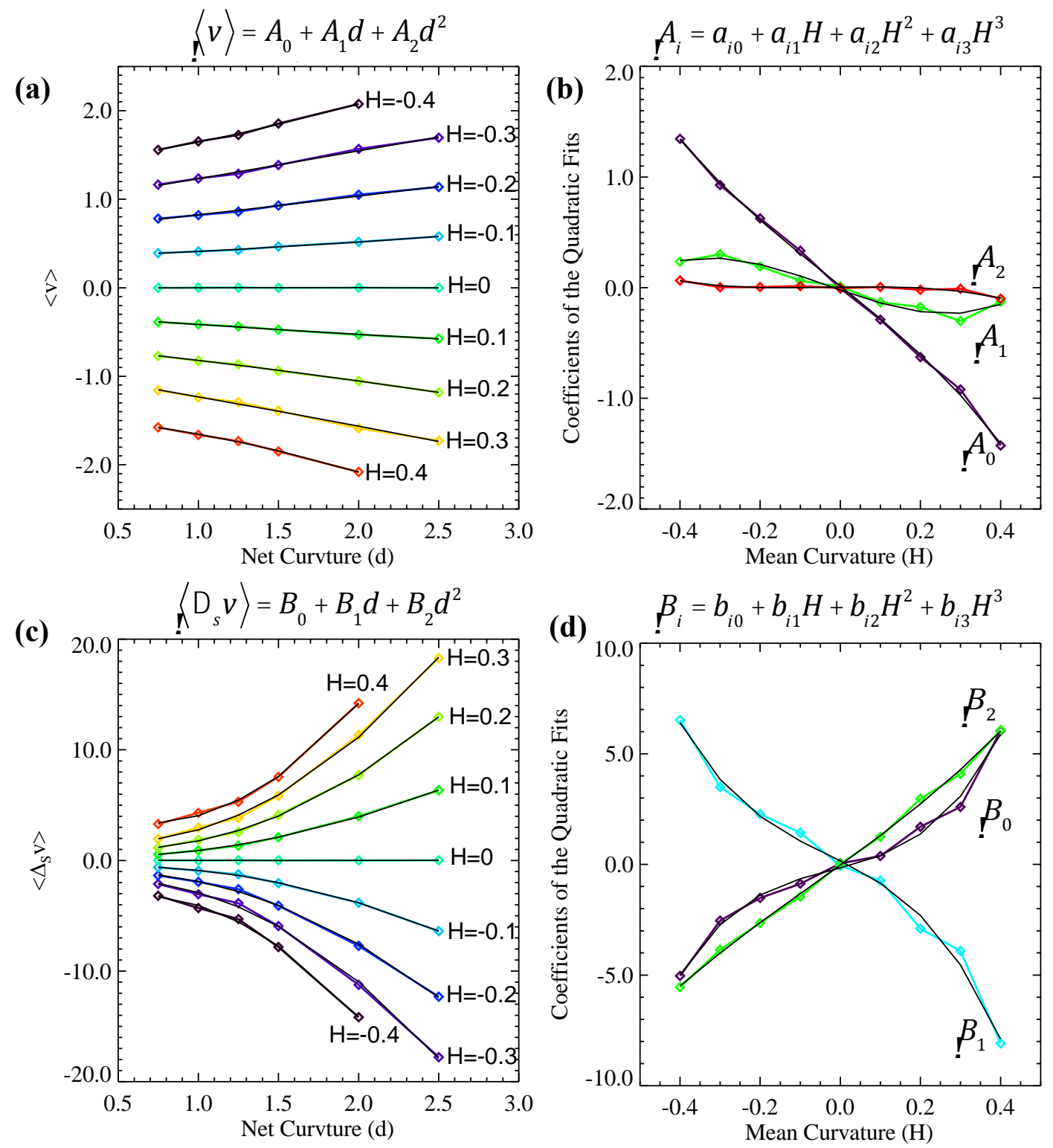

Figure 7. (a) Values of $\langle v\rangle_{H, d}$, calculated from the simulation data, as a function of $d$ for different values of $H$ for the 50:50 $\mathrm{CH}$ structure are drawn with the colored diamond symbols (connected by solid colored lines). Each color represents a given $H$ value. The quadratic fit, in the form of $A_{0}(H)+A_{1}(H) d+A_{2}(H) d^{2}$, for each set of points for a given $H$ value is overplotted with a solid black curve. (b) Values of the coefficients, $A_{0}(H), A_{1}(H)$ and $A_{2}(H)$, as functions of $H$ are drawn with colored diamond symbols (connected by solid colored lines). The cubic polynomial fit, in the form of $A_{i}=a_{i 0}+a_{i 1} H+a_{i 2} H^{2}+a_{i 3} H^{3}$ with $i=0,1$ and 2 , for each coefficient is overplotted with a solid black curve. Subfigures (c) and (d) are similar to (a) and (b) 
but for the average surface Laplacian of interfacial velocity, $\left\langle{ }_{S} v\right\rangle_{H, d}$, and the coefficients of its quadratic fit, $B_{0}(H), B_{1}(H)$ and $B_{2}(H)$.

Fig. 7a shows the data sets of $\langle v\rangle_{H, d}$ versus $d$ for different values of $H$ for the 50:50 $\mathrm{CH}$ structure drawn with solid diamonds, where each color represents a given $H$ value. A quadratic fit in the form of

$$
\langle v\rangle_{H, d}=A_{0}(H)+A_{1}(H) d+A_{2}(H) d^{2}
$$

is applied on each set of $\langle v\rangle_{H, d}$ points for a given $H$ value and is shown with a solid black curve on the same plot. To find the expressions for the coefficients, $A_{0}(H), A_{1}(H)$ and $A_{2}(H)$, in terms of $H$, we also plotted the values of coefficients versus $H$ as colored diamonds. A cubic polynomial fit in the form of

$$
A_{i}=a_{i 0}+a_{i 1} H+a_{i 2} H^{2}+a_{i 3} H^{3}
$$

where $i=0,1$ and 2 , is applied to each coefficient and is shown with a solid black curve (Fig. 7b). The two numerical fits, Eqs. (13) and (14), result in the expression for $\langle v\rangle_{H, d}$ in the form of a combination of a quadratic function of $d$ and a cubic function of $H$. The values of the fit parameters $a_{i 0}, a_{i 1}, a_{i 2}$, and $a_{i 3}$ in Eq. (14) are shown in the appendix.

The same approach was used to obtain the expression for $\left\langle{ }_{S} v\right\rangle_{H, d}$. The plots of data points and their numerical fits are shown in Fig. 7c and d. Similar to the expression for $\langle v\rangle_{H, d}$, the 
expression for $\left\langle{ }_{S} V\right\rangle_{H, d}$ is a combination of a quadratic function of $d$ and a cubic function of $H$.

The values of the fit parameters $b_{i 0}, b_{i 1}, b_{i 2}$, and $b_{i 3}$ are shown in the appendix. We find that $\langle v\rangle_{H, d}$ and $\left\langle{ }_{S} v\right\rangle_{H, d}$ for the 40:60 and 30:70 $\mathrm{CH}$ structures also have quadratic and cubic dependencies on $d$ and $H$, respectively (not shown).

The expressions for $\langle v\rangle_{H, d}$ and $\left\langle{ }_{S} v\right\rangle_{H, d}$ are substituted into Eq. (12) to obtain the expression for $\langle D H / D t\rangle_{H, d}$ as a function of the local curvatures: $H$ and $d$. The predicted values of $\langle D H / D t\rangle_{H, d}$ based on the expressions of $\langle v\rangle_{H, d}$ and $\left\langle{ }_{S} v\right\rangle_{H, d}$ are compared with $\langle D H / D t\rangle_{H, d}$ directly calculated from $D H / D t$ at all interfaces with given values of $H$ and $d$ in Fig. 8. The calculated $\langle D H / D t\rangle_{H, d}$ is represented as solid colored curves while the predicted $\langle D H / D t\rangle_{H, d}$ is represented as dashed colored curves. The predicted $\langle D H / D t\rangle_{H, d}$ agrees well with the calculated $\langle D H / D t\rangle_{H, d}$

The plot of $\langle D H / D t\rangle_{H, d}$ for the 50:50 CH structure, Fig. 8a, shows that $H$ of interfaces with low net curvature, $d \lesssim 1.2$, evolves toward the average mean curvature, $\bar{H}=0$, while $H$ of interfaces with larger net curvature, $d \gtrsim 1.2$, diverges from $\bar{H}$. For example, the red curve corresponding to $H=0.4$ is negative for $d \lesssim 1.2$, indicating $H$ is evolving toward $\bar{H}=0$, while it becomes positive for $d \gtrsim 1.2$, which would result in increasing $H$ further. This finding demonstrates that interfaces with low net curvature are evolving to reduce the local mean curvature during coarsening. On the other hand, interfaces with large net curvature appear to break the symmetry 
of the saddle-shape even when $|H|$ is not large $(\mid H \gtrsim 0.1)$, which can be understood as a part of the process leading to a topological singularity. The same trend is observed for the plot of $\langle D H / D t\rangle_{H, d}$ of the 40:60 CH structure, Fig. 8b, which has $\bar{H}=0.2$.

In the case of the nonbicontinuous 30:70 $\mathrm{CH}$ structure, the evolution of mean curvature is more complex, as indicated by the plot of $\langle D H / D t\rangle_{H, d}$ shown in Fig. 8c. We find that $H$ of interfaces with $H \gtrsim \bar{H}=-1.1$ and $d \lesssim 1.2$ evolves toward $\bar{H}$, while $H$ of interfaces with $H \gtrsim \bar{H}$ and $d \gtrsim 1.2$ diverges from $\bar{H}$. This is consistent with the results for bicontinuous structures. Indeed a sizable portion of interfaces with $H \gtrsim \bar{H}$ of the $30: 70 \mathrm{CH}$ structure are saddle-shaped, like those of the bicontinuous structures, as can be observed in the ISD in Fig. 3(c.1). However, this trend in the evolution of $H$ gradually disappears for interfaces with $H \lesssim \bar{H}$. For interfaces with $H \lesssim \bar{H}$, which are mostly of isolated domains that have elliptical geometry, we find that $|H|$ increases on average, which represents dissolution of high-curvature interfaces. Thus, the observation that low net curvature regions evolve toward reducing the difference between the average mean curvature and the local mean curvature, while high net curvature regions evolve in the opposite direction, may be universal not only to bicontinuous structures but also to hyperbolic interfaces within transient structures that are similar to bicontinuous structures.

The results obtained from the semi-analytical approach demonstrate that the average rate of change of curvatures can be predicted based on the local curvatures, even though the interfaces are interacting by long-range diffusion fields. Furthermore, as discussed in the introduction, an analytical expression for the growth rate of a particle, $d R / d t$, as a function of its radius, $R$, was a critical input in deriving the time-independent particle size distribution and the coarsening law. 
As in the LSW theory, the expression for the average rate of change of curvature we have obtained here can be used to solve for the time-independent interfacial shape distribution using the continuity equation. This should be the next step in the development of the theory of coarsening of complex structures.

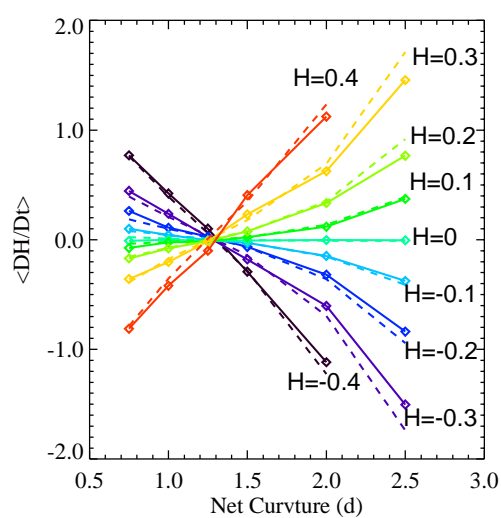

(a)

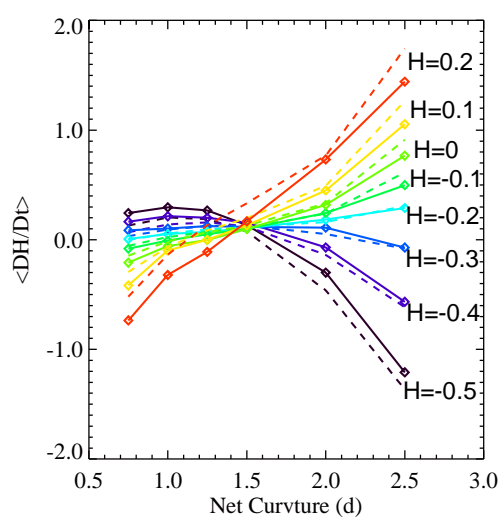

(b)

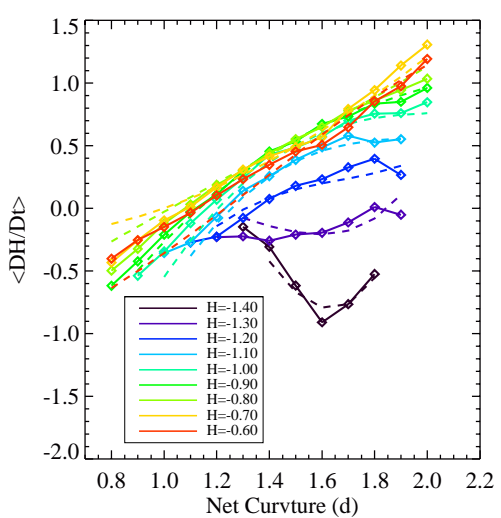

(c)

Figure 8. The predicted values of $\langle D H / D t\rangle_{H, d}$ based on Eq. (12) for the $\mathrm{CH}$ structure with volume fractions of (a) 50:50, (b) 40:60, and (c) 30:70 are shown by dashed colored curves, along with the calculated values of $\langle D H / D t\rangle_{H, d}$ given by the colored diamond symbol, connected by solid colored curves.

\section{Conclusion}

In this paper, we have examined the correlations between interfacial velocity and static local properties, and developed a semi-analytical approach to predicting curvature evolution of complex structures undergoing coarsening following spinodal decomposition. Three simulated $\mathrm{CH}$ structures are generated using phase-field simulations with different volume fractions that resulted in two bicontinuous structures and one nonbicontinuous structure. The level-set smoothing method is employed to ensure that various interfacial quantities used in the analysis are accurately calculated. Below are the key findings presented in this paper. 
- The probability contour maps have shown that there exists a correlation between $v$ and $H$; such correlation has been observed for dendritic mixtures of $\mathrm{Al}-\mathrm{Cu}$ alloy [42]. We also find that average interfacial velocity is described by a polynomial of the mean curvature and the net curvature.

- We also identified a correlation between $v$ and ${ }_{S} H$; such correlation has not yet been examined in experiments to date.

- We find that the distribution of normal velocities of interfaces with the same local principal curvatures fits a Gaussian function well, independent of the local principal curvatures and the volume fractions of the structures. The origin of the Gaussian distribution is still not understood and requires further investigation.

- We have successfully developed a semi-analytical approach that can be employed to predict the evolution of mean curvature, which represents a significant advance in the development of the theory of coarsening of complex structures.

\section{Acknowledgments}

Authors are grateful for the support from the Department of Energy Office of Basic Energy Science (Grant No. DE-FG02-99ER45782). The computational resources were provided by the Extreme Science and Engineering Discovery Environment (XSEDE), which is supported by National Science Foundation grant number OCI-1053575, under allocation No. TG-DMR110007, as well as the University of Michigan Advanced Research Computing.

\section{Appendix}


7.1 Fit parameters for $\langle v\rangle_{H}$ and $\langle v\rangle_{S^{H}}$ in Sec. 4.1

The parameters for the cubic polynomial fit in the form of

$$
\langle v\rangle=c_{0}+c_{1}+c_{2}{ }^{2}+c_{3}{ }^{3},
$$

where $=H$ or $={ }_{s} H$, to $\langle v\rangle_{H}$ and $\langle v\rangle_{s^{H}}$ are presented in Table 2 for the three $\mathrm{CH}$ structures presented Fig. 3(a.2)-(c.2) and (a.3)-(c.3). Note that, in the evaluation of the averages, velocity values that do not fall within \pm 8 of the approximate average velocities were deemed outliers and were removed from the calculation of the $\langle v\rangle_{H}$ and $\langle v\rangle_{s^{H}}$. These outliers with physically unrealistic values arise from numerical errors caused by unresolved high curvature regions approaching or evolving away from topological singularities. Such data amounts to only about $1 \%$ of the total statistics. The curvature outliers do not influence the polynomial fits of $\langle v\rangle_{H}$ and $\langle v\rangle_{S^{H}}$ since we apply the fit over the $H$ range of $[-2,2]([-1,3]$ for the $30: 70 \mathrm{CH}$ structure only) and ${ }_{S} H$ range of [-6,6].

\begin{tabular}{|c|c|c|c|c|c|}
\hline \multirow{2}{*}{ Structure } & $\begin{array}{c}\text { Quantity } \\
\text { Fitted }\end{array}$ & $c_{0}$ & $c_{1}$ & $c_{2}$ & $c_{3}$ \\
\hline $\begin{array}{c}50: 50 \\
\text { CH Structure }\end{array}$ & $\langle v\rangle_{H}$ & $2.0 E-2$ & $4.7 E 0$ & $-5.1 E-2$ & $7.4 E-1$ \\
\cline { 2 - 6 } & $\langle v\rangle_{s^{H}}$ & $-1.9 E-3$ & $8.4 E-1$ & $4.8 E-4$ & $-6.5 E-3$ \\
\hline $40: 60$ & $\langle v\rangle_{H}$ & $-1.2 E 0$ & $4.0 E 0$ & $-3.3 E-1$ & $6.1 E-1$ \\
\hline
\end{tabular}




\begin{tabular}{|c|c|c|c|c|c|}
\hline CH Structure & $\langle v\rangle_{S^{H}}$ & $-2.0 E-2$ & $6.8 E-1$ & $2.8 E-3$ & $-4.6 E-3$ \\
\hline $\begin{array}{c}30: 70 \\
\text { CH Structure }\end{array}$ & $\langle v\rangle_{H}$ & $-2.1 E 0$ & $3.0 E 0$ & $-1.0 E 0$ & $3.0 E-1$ \\
\cline { 2 - 6 } & $\langle v\rangle_{S^{H}}$ & $-6.2 E-2$ & $3.0 E-1$ & $4.6 E-3$ & $-7.4 E-5$ \\
\hline
\end{tabular}

Table 2. The parameters of the cubic polynomial fit of $\langle v\rangle_{H}$ and $\langle v\rangle_{s^{H}}$ for the three $\mathrm{CH}$ structures.

7.2 Fit parameters for $\langle v\rangle_{H, d}$ and $\left\langle{ }_{s} v\right\rangle_{H, d}$ in Sec. 4.4.

In Sec 4.4, it was shown that $\langle v\rangle_{H, d}$ and $\left\langle{ }_{S} v\right\rangle_{H, d}$ can be fitted by quadratic functions in terms of net curvature, $d$. It was further shown that the fit coefficients are well represented by the cubic functions in terms of the mean curvature, $H$. In Table 3, the parameters $a_{i 0}, a_{i 1}, a_{i 2}$, and $a_{i 3}$ are for $\langle v\rangle_{H, d}$ while the parameters $b_{i 0}, b_{i 1}, b_{i 2}$, and $b_{i 3}$ are for $\left\langle{ }_{s} v\right\rangle_{H, d}$, where $i=0,1$ and 2 indicate the powers of $d$ in the quadratic fit of $\langle v\rangle_{H, d}$ and $\left\langle{ }_{s} v\right\rangle_{H, d}$. For the calculation of $\langle v\rangle_{H, d}$, we have used the same range of velocity values as that used to calculate $\langle v\rangle_{H}$ in Sec. 4.1. For the calculation of $\left\langle{ }_{s} V\right\rangle_{H, d}$ and $\langle D H / D t\rangle_{H, d}$ (directly calculated quantity which is compared with the predicted $\langle D H / D t\rangle_{H, d}$ in Fig. 8), we have excluded ${ }_{s} V$ and $D H / D t$ values outside the range of $[-80,80]$ and $[-30,30]$, respectively, which arise from numerical errors and constitute about $1 \%$ of the entire data.

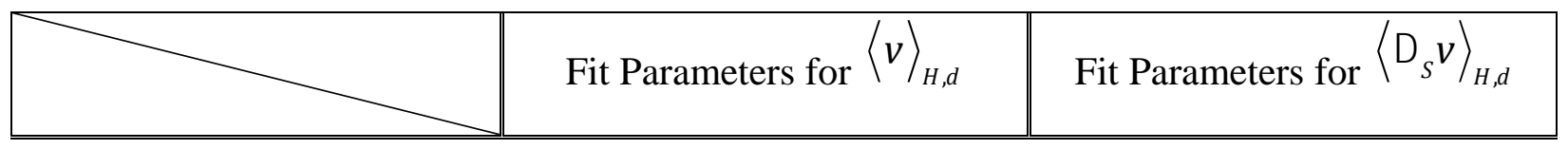




\begin{tabular}{|c|c|c|c|c|c|c|c|c|c|}
\hline Structure & $\begin{array}{l}\text { Coefficients of the } \\
\text { Quadratic Fit }(i)\end{array}$ & $a_{i 0}$ & $a_{i 1}$ & $a_{i 2}$ & $a_{i 3}$ & $b_{i 0}$ & $b_{i 1}$ & $b_{i 2}$ & $b_{i 3}$ \\
\hline \multirow{3}{*}{$\begin{array}{c}50: 50 \\
\mathrm{CH} \\
\text { Structure }\end{array}$} & 0 & $1.6 E-2$ & $-2.9 E 0$ & $-3.1 E-1$ & $-3.5 E 0$ & $-2.3 E 0$ & $1.3 E 1$ & $2.9 E 1$ & $1.8 E 1$ \\
\hline & 1 & $-2.0 E-2$ & $-1.3 E 0$ & $4.2 E-1$ & $4.8 E 0$ & $-3.8 E 0$ & $-1.8 E 1$ & $-3.1 E 1$ & $-4.7 E 0$ \\
\hline & 2 & $4.7 E-3$ & $5.4 E-2$ & $-1.3 E-1$ & $-1.5 E 0$ & $3.5 E 0$ & $1.4 E 1$ & $7.1 E 0$ & $-6.2 E 0$ \\
\hline \multirow{3}{*}{$\begin{array}{c}\text { 40:60 } \\
\text { CH } \\
\text { Structure }\end{array}$} & 0 & $-8.2 E-1$ & $-3.1 E 0$ & $-1.5 E 0$ & $-2.9 E-1$ & $2.1 E 0$ & $1.1 E 1$ & $2.5 E 1$ & $1.5 E 1$ \\
\hline & 1 & $-8.7 E-2$ & $-2.6 E-1$ & $1.4 E 0$ & $-3.9 E-1$ & $-3.7 E 0$ & $-1.7 E 1$ & $-3.0 E 1$ & $-7.1 E 0$ \\
\hline & 2 & $-4.9 E-2$ & $-1.9 E-1$ & $-3.0 E-1$ & $3.5 E-1$ & $3.5 E 0$ & $1.4 E 1$ & $6.9 E 0$ & $-5.0 E 0$ \\
\hline \multirow{3}{*}{$\begin{array}{c}30: 70 \\
\mathrm{CH} \\
\text { Structure }\end{array}$} & 0 & $1.0 E 1$ & $4.0 E 1$ & $4.8 E 1$ & $2.0 E 1$ & $2.5 E 2$ & $9.3 E 2$ & $1.1 E 3$ & $4.0 E 2$ \\
\hline & 1 & $-1.4 E 1$ & $-4.9 E 1$ & $-5.7 E 1$ & $-2.3 E 1$ & $-3.1 E 2$ & $-1.1 E 3$ & $-1.4 E 3$ & $-5.1 E 2$ \\
\hline & 2 & $4.4 E 0$ & $1.5 E 1$ & $1.7 E 1$ & $6.8 E 0$ & $9.7 E 1$ & $3.5 E 2$ & $4.2 E 2$ & $1.6 E 2$ \\
\hline
\end{tabular}

Table 3. The parameters of the cubic polynomial fit of $\langle v\rangle_{H, d}$ and $\left\langle{ }_{s} V\right\rangle_{H, d}$ for the three $\mathrm{CH}$ structures.

\subsection{Accuracy of the calculation of surface Laplacian of the mean curvature}

The roughness in the color that appears in Fig. 4(a.3)-(c.3) is the result of the grid effect that manifests itself when high-order derivatives are calculated at interfaces with insufficient interfacial resolution. The level-set smoothing method applied to the microstructural data is designed to smooth up to the second-order derivatives required in the calculation of curvatures, as evidenced by the smooth $H$ profiles in Fig. 4(a.1)-(c.1). Since ${ }_{s} H$ requires calculation of fourth-order derivatives across the interfaces, the level-set smoothing method cannot fully 
eliminate the numerical noise associated with the calculation of ${ }_{s} H$. Thus, the quality of data from the calculation of ${ }_{s} H$ is slightly affected. Nevertheless, the isosurfaces of ${ }_{s} H$ and the probability contour maps of $P_{2}\left({ }_{s} H, v\right)$, Fig. 3(a.3)-(c.3), are essential in highlighting the importance of nearby interfacial curvatures on the interfacial velocity.

\section{References}

[1] H.A. Calderon, P.W. Voorhees, J.L. Murray, G. Kostorz, Ostwald ripening in concentrated alloys, Acta Metall. Mater. 42(3) (1994) 991-1000.

[2] A.J. Ardell, R.B. Nicholson, Coarsening of gamma' in Ni-Al alloys, J. Phys. Chem. Solids 27(11-1) (1966) 1793-1804.

[3] T.M. Pollock, A.S. Argon, Directional coarsening of nickel-base single-crystals with highvolume fractions of coherent precipitates, Acta Metall. Mater. 42(6) (1994) 1859-1874.

[4] S. Wang, E.A. Holm, J. Suni, M.H. Alvi, P.N. Kalu, A.D. Rollett, Modeling the recrystallized grain size in single phase materials, Acta Mater. 59(10) (2011) 3872-3882.

[5] T. Hashimoto, M. Takenaka, T. Izumitani, Spontaneous pinning of domain growth during spinodal decomposition of off-critical polymer mixtures, J. Chem. Phys. 97(1) (1992) 679-689.

[6] S.W. Song, J.M. Torkelson, Coarsening effects on microstructure formation in isopycnic polymer-solutions and membranes produced via thermally-induced phase-separation, Macromolecules 27(22) (1994) 6389-6397.

[7] G. Krausch, C.A. Dai, E.J. Kramer, J.F. Marko, F.S. Bates, Interference of spinodal waves in thin polymer-films, Macromolecules 26(21) (1993) 5566-5571.

[8] P. Wiltzius, A. Cumming, Domain growth and wetting in polymer mixtures, Phys. Rev. Lett. 66(23) (1991) 3000-3003.

[9] J. Erlebacher, I. McCue, Geometric characterization of nanoporous metals, Acta Mater. 60(17) (2012) 6164-6174.

[10] Y.C.K. Chen, Y.S. Chu, J. Yi, I. McNulty, Q. Shen, P.W. Voorhees, D.C. Dunand, Morphological and topological analysis of coarsened nanoporous gold by $\mathrm{x}$-ray nanotomography, Appl. Phys. Lett. 96(4) (2010) 043122.

[11] O. Kienzle, F. Ernst, M. Ruhle, O.G. Schmidt, K. Eberl, Germanium "quantum dots" embedded in silicon: Quantitative study of self-alignment and coarsening, Appl. Phys. Lett. 74(2) (1999) 269-271.

[12] F. Liu, A.H. Li, M.G. Lagally, Self-assembly of two-dimensional islands via strainmediated coarsening, Phys. Rev. Lett. 87(12) (2001) 126103.

[13] F.M. Ross, J. Tersoff, R.M. Tromp, Coarsening of self-assembled Ge quantum dots on Si(001), Phys. Rev. Lett. 80(5) (1998) 984-987.

[14] J. Miyake, M.E. Fine, Electrical-conductivity versus strength in a precipitation hardened alloy, Acta Metall. Mater. 40(4) (1992) 733-741. 
[15] H.-Y. Chen, H.-C. Yu, J.S. Cronin, J.R. Wilson, S.A. Barnett, K. Thornton, Simulation of coarsening in three-phase solid oxide fuel cell anodes, J. Power Sources 196(3) (2011) 13331337.

[16] R. Davis, F. Abdeljawad, J. Lillibridge, M. Haataja, Phase wettability and microstructural evolution in solid oxide fuel cell anode materials, Acta Mater. 78 (2014) 271-281.

[17] P. Tanasini, M. Cannarozzo, P. Costamagna, A. Faes, J. Van Herle, A. Hessler-Wyser, C. Comninellis, Experimental and theoretical investigation of degradation mechanisms by particle coarsening in SOFC electrodes, Fuel Cells 9(5) (2009) 740-752.

[18] K.R. Mangipudi, E. Epler, C.A. Volkert, Topology-dependent scaling laws for the stiffness and strength of nanoporous gold, Acta Mater. 119 (2016) 115-122.

[19] E.T. Lilleodden, Private communication, September 24, 2016.

[20] P.W. Voorhees, The theory of Ostwald ripening, J. Stat. Phys. 38(1-2) (1985) 231-252.

[21] A.D. Brailsford, P. Wynblatt, Dependence of Ostwald ripening kinetics on particle-volume fraction, Acta Metall. 27(3) (1979) 489-497.

[22] J.A. Marqusee, J. Ross, Theory of Ostwald ripening - competitive growth and its dependence on volume fraction, J. Chem. Phys. 80(1) (1984) 536-543.

[23] I.M. Lifshitz, V.V. Slyozov, The kinetics of precipiration from supersaturated solid solutions, J. Phys. Chem. Solids 19(1-2) (1961) 35-50.

[24] C. Wagner, Theorie der alterung von niederschlagen durch umlosen (Ostwald-reifung), Z. Elektrochem. 65(7-8) (1961) 581-591.

[25] P.W. Voorhees, Ostwald ripening of 2-phase mixtures, Annu. Rev. Mater. Sci. 22 (1992) 197-215.

[26] A. Baldan, Review progress in Ostwald ripening theories and their applications to nickelbase superalloys - Part I: Ostwald ripening theories, J. Mater. Sci. 37(11) (2002) 2171-2202.

[27] L. Ratke, P.W. Voorhees, Growth and coarsening: Ostwald ripening in material processing, Springer, Berlin, 2002.

[28] K.P. Young, D.H. Kirkwood, Dendrite arm spacings of aluminum-copper alloys solidified under steady-state conditions, Metall. Trans. A 6(1) (1975) 197-205.

[29] M. Kahlweit, On ageing of dendrites, Scripta Metall. 2(5) (1968) 251-254.

[30] T.Z. Kattamis, J.C. Coughlin, M.C. Flemings, Influence of coarsening on dendrite arm spacing of aluminum-copper alloys, Trans. Metall. AIME 239(10) (1967) 1504-1511.

[31] J.J. Reeves, T.Z. Kattamis, Model for isothermal dendritic coarsening, Scripta Metall. 5(3) (1971) 223-230.

[32] C.-L. Park, P.W. Voorhees, K. Thornton, Evolution of interfacial curvatures of a bicontinuous structure generated via nonconserved dynamics, Acta Mater. 90 (2015) 182-193.

[33] M. Hillert, On theory of normal and abnormal grain growth, Acta Metall. 13(3) (1965) 227 238.

[34] J.E. Taylor, J.W. Cahn, C.A. Handwerker, Overview No. 98 I-Geometric models of crystal growth, Acta Metall. Mater. 40(7) (1992) 1443-1474.

[35] S.M. Allen, J.W. Cahn, Microscopic theory for antiphase boundary motion and its application to antiphase domain coarsening, Acta Metall. 27(6) (1979) 1085-1095.

[36] L.Q. Chen, Phase-field models for microstructure evolution, Annu. Rev. Mater. Res. 32 (2002) 113-140.

[37] A. Karma, W.J. Rappel, Phase-field method for computationally efficient modeling of solidification with arbitrary interface kinetics, Phys. Rev. E 53(4) (1996) R3017-R3020. 
[38] J.A. Warren, W.J. Boettinger, Prediction of dendritic growth and microsegregation patterns in a binary alloy using the phase-field method, Acta Metall. Mater. 43(2) (1995) 689-703.

[39] J.W. Cahn, On spinodal decomposition, Acta Metall. 9(9) (1961) 795-801.

[40] J.W. Cahn, J.E. Hilliard, Free energy of a nonuniform system .I. interfacial free energy, J. Chem. Phys. 28(2) (1958) 258-267.

[41] Y. Kwon, K. Thornton, P.W. Voorhees, Morphology and topology in coarsening of domains via non-conserved and conserved dynamics, Philos. Mag. 90(1-4) (2010) 317-335.

[42] J.L. Fife, J.W. Gibbs, E.B. Gulsoy, C.-L. Park, K. Thornton, P.W. Voorhees, The dynamics of interfaces during coarsening in solid-liquid systems, Acta Mater. 70 (2014) 66-78.

[43] D.A. Drew, Evolution of geometric statistics, SIAM J. Appl. Math. 50(3) (1990) 649-666.

[44] C.-L. Park, P.W. Voorhees, K. Thornton, Application of the level-set method to the analysis of an evolving microstructure, Comput. Mater. Sci. 85 (2014) 46-58.

[45] J.J. Xu, H.K. Zhao, An Eulerian formulation for solving partial differential equations along a moving interface, J. Sci. Comput. 19(1-3) (2003) 573-594.

[46] J.A. Sethian, Level set methods and fast marching methods: evolving interfaces in computational geometry, fluid mechanics, computer vision, and materials science, Second ed., Cambridge University Press 1999.

[47] R. Mendoza, J. Alkemper, P.W. Voorhees, The morphological evolution of dendritic microstructures during coarsening, Metall. Mater. Trans. A 34(3) (2003) 481-489.

[48] W.W. Mullins, Theory of thermal grooving, J. Appl. Phys. 28(3) (1957) 333-339.

[49] M.B. Wilk, R. Gnanadesikan, Probability plotting methods for the analysis of data, Biometrika 55(1) (1968) 1-17. 


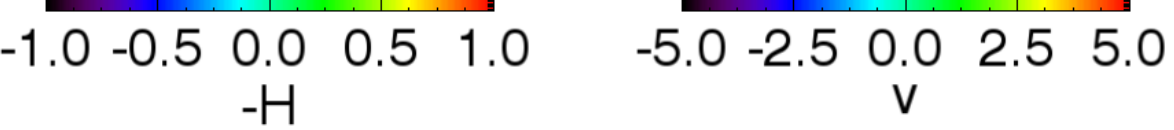

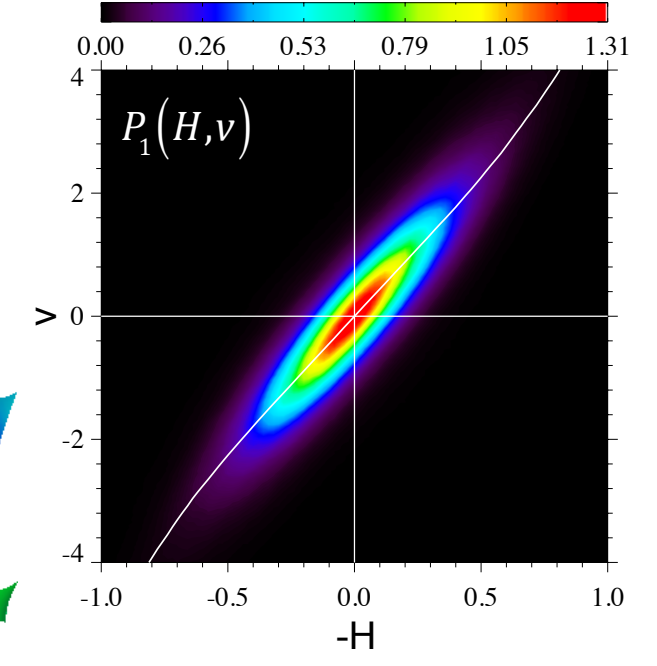

